\title{
Use of rectal mucosal grafts in substitution urethroplasty: an early series
}

\author{
M. Francesca Monn ${ }^{1}$, Joshua A. Waters ${ }^{2}$, Matthew J. Mellon ${ }^{1}$ \\ ${ }^{1}$ Department of Urology, ${ }^{2}$ Department of Surgery, Indiana University School of Medicine, Indianapolis, IN, USA \\ Contributions: (I) Conception and design: MJ Mellon, JA Waters; (II) Administrative support: None; (III) Provision of study materials or patients: MJ \\ Mellon, JA Waters; (IV) Collection and assembly of data: MJ Mellon, JA Waters; (V) Data analysis and interpretation: MJ Mellon; (VI) Manuscript \\ writing: All authors; (VII) Final approval of manuscript: All authors. \\ Correspondence to: Matthew J. Mellon, MD. 1801 Senate St. Suite 220, Indianapolis, IN 46202, USA. Email: mjmellon@iupui.edu.
}

\begin{abstract}
Background: To evaluate the feasibility of use of rectal mucosal grafts for augmentation urethroplasty.
Methods: A series of five patients who underwent rectal mucosal graft urethroplasty for urethral stricture disease were identified. Descriptive statistics were used to describe these patients. Primary endpoints were recurrence of stricture and perioperative morbidity.

Results: Five patients underwent rectal mucosal graft augmentation urethroplasty. Four had a history of prior buccal mucosal graft (BMG) urethroplasty and one had a history of head and neck cancer. Rectal mucosa was noted to be thinner and required more tailoring than buccal mucosa. All patients had patent urethras at time of postoperative retrograde urethrogram. A small diverticulum was noted in one patient with no further sequelae. No complications from rectal mucosal graft harvest were noted. All patients with prior buccal grafting subjectively preferred the rectal graft due to fewer side effects. Subjectively, patients with prior buccal grafts preferred the post-operative recovery following rectal mucosal graft urethroplasty.

Conclusions: Rectal mucosal graft augmentation urethroplasty is a safe alternative in patients with contraindications to buccal grafting with limited morbidity.
\end{abstract}

Keywords: Rectal mucosal graft; substitution urethroplasty; transanal endoscopic microsurgery (TEM); urethral stricture

Submitted Sep 17, 2018. Accepted for publication Oct 22, 2018.

doi: $10.21037 /$ tau.2018.10.12

View this article at: http://dx.doi.org/10.21037/tau.2018.10.12

\section{Introduction}

Substitution or augment urethroplasty is the gold standard for long urethral strictures. Multiple graft substrates have been used over the years including buccal, lingual, penile, and scrotal grafts although buccal grafts have remained the most widely used. Reports of using bowel mucosa for substitution urethroplasty date back to 1994 when Lebret et al. reported using appendiceal mucosa $(1,2)$. Xu et al. first reported using tubularized colonic mucosa in 2003 for the repair of recurrent urethral strictures $(3,4)$. Most recently Palmer et al. reported on endoscopic rectal mucosal graft harvest with subsequent rectal mucosal graft substitution urethroplasties with promising early results (5).
Rectal mucosal grafts provide an exciting alternative to buccal mucosa as harvested segments of rectal mucosa can measure up to $8 \mathrm{~cm}$ in diameter. Additionally, histologic studies of colonic mucosa exposed previously to urine demonstrated the ability of the colonic mucosa to adapt as a non-secreting urothelium (2). In Palmer et al.'s early report of using rectal mucosal substitution grafts, patients selected for the study were those with long urethral strictures, primarily secondary to lichen sclerosus, with median stricture length of $13.5 \mathrm{~cm}$ (5). In the current study we chose to evaluate the use of rectal mucosal substitution grafts in patients with prior buccal mucosal grafts (BMGs) or head and neck radiation, thus precluding use of a BMG, to evaluate its use in a setting wherein buccal mucosa is not a graft option. 

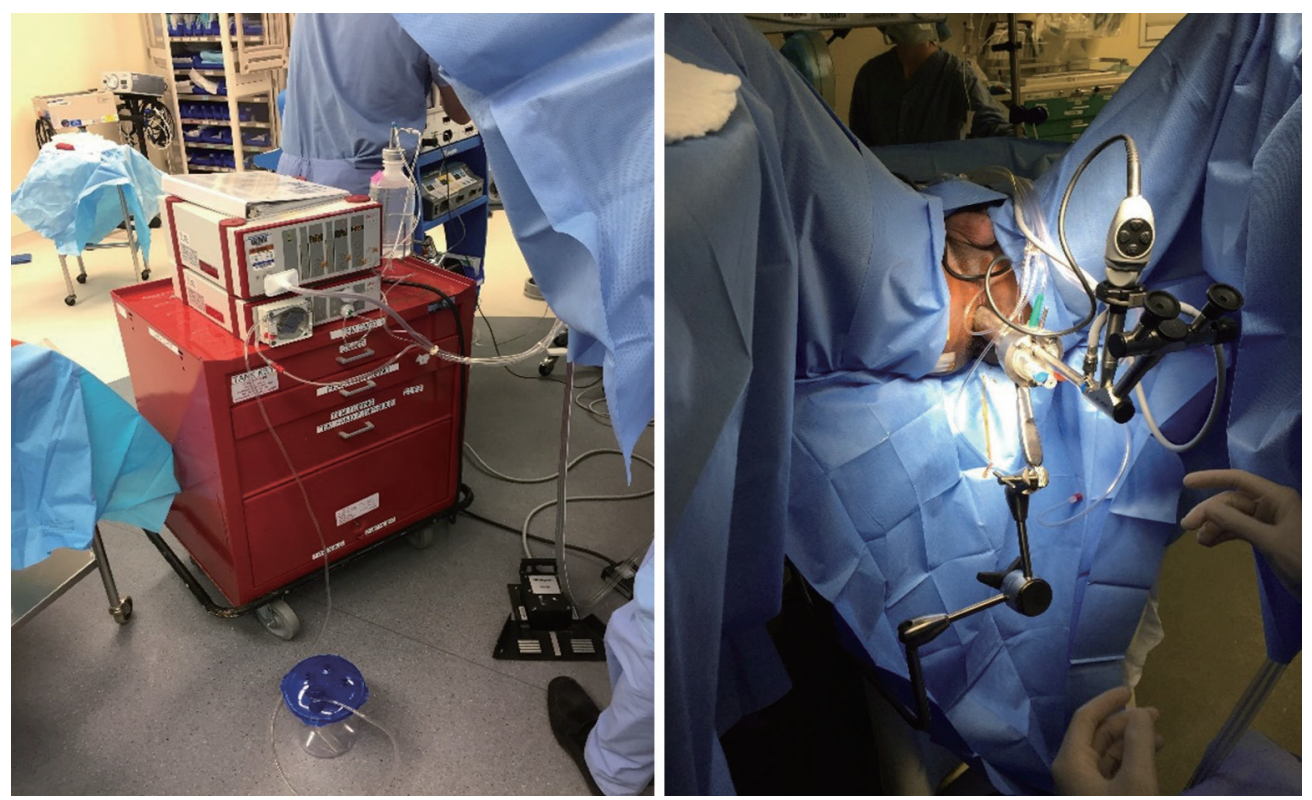

Figure 1 TEM rectoscope. TEM, transanal endoscopic microsurgery.

\section{Methods}

\section{Patient selection}

All patients undergoing rectal mucosal graft substitution urethroplasty from May 2016 to June 2017 were identified from an institutional database. All surgeries were performed by MJ Mellon and JA Waters. Patients were selected for rectal mucosal graft substitution urethroplasty in the setting of recurrent stricture following a BMG urethroplasty or having a long stricture with a contraindication to undergoing BMG urethroplasty. The novel nature of the technique was explained to all patients who expressed understanding and willingness to proceed.

\section{Preoperative evaluation, surgical technique, and postoperative management}

Patients selected for a rectal mucosal graft substitution urethroplasty were sent for further evaluation by the colorectal surgeon (JA Waters). All patients underwent colonoscopy to ensure no abnormalities in their lower gastrointestinal tract and to evaluate the mucosa. Once approved patients were scheduled for surgery using the transanal endoscopic microsurgery (TEM) rectoscope. Bowel prep was not used preoperatively.

To begin the surgery, patients were positioned in dorsal lithotomy. The anal sphincter was dilated after application of local anesthetic. The rectoscope was then introduced and the rectal lumen insufflated. The harvest site was measured and marked. The distal flap was initiated just proximal to the hemorrhoidal complex in the posterior midline. $1 \%$ lidocaine with epinephrine was infiltrate within the submucosal plane to allow for lift of the flap. The rectal mucosal dissection begins distally and the graft was rolled proximally the desired length. The resection bed was left intact without closure for healing by secondary intent. Figure 1 demonstrates the TEM rectoscope and patient positioning.

After performing the perineal dissection and isolating the strictured portion of the urethra, the rectal mucosa graft (Figure 2) was tailored and trimmed for the specific graft length needed (MJ Mellon). The remainder of the substitution urethroplasty was performed in standard fashion, and the rectal mucosal graft was laid in as a ventral onlay graft as is practice at our institution.

Post-operatively patients were discharged to home with a catheter in place for 3 weeks with follow-up scheduled with a peri-catheter retrograde urethrogram. No leaks were noted and all catheters were removed at the post-operative visit. Patients were brought back to clinic at intervals of 3-6 months with uroflow and post void residual. Retrograde urethrogram was obtained at approximately 6 months postoperatively to ensure graft patency and are presented herein. 


\section{Statistical analysis}

Descriptive statistics were used to evaluate patients undergoing rectal mucosal graft substitution urethroplasty. The Indiana University School of Medicine Institutional Review Board exempt status was granted for the conduct of the study. As this was performed as a retrospective review of outcomes, patients did not undergo a separate consent from the procedural consent prior to surgery.

\section{Results}

Five patients were identified for inclusion in the study with median follow-up time of 13.5 months (range, 12.5-25.5 months) (Table 1). Four patients had prior BMG urethroplasties and one had a history of undergoing radiation for head and neck cancer. Average (SD) age at time of surgery was 52.4 (13.1). Average (SD) stricture length was $4.4(0.5) \mathrm{cm}$. Stricture location was bulbar urethra for four and pendulous urethra for the fifth patient. Patent urethras were demonstrated in all five patients

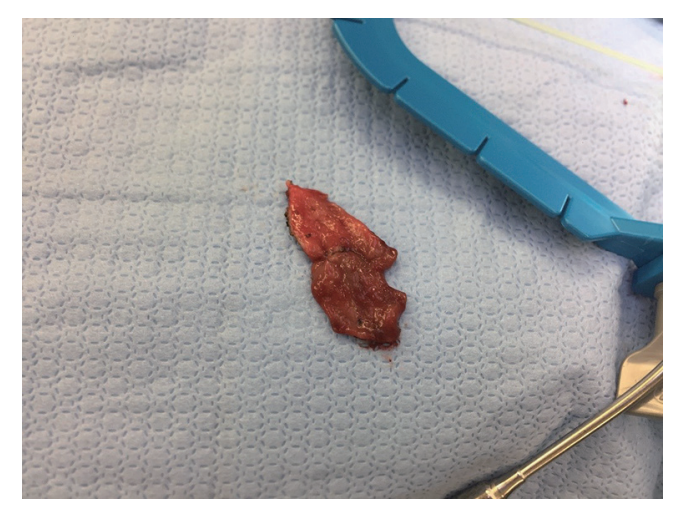

Figure 2 Rectal mucosal graft. on post-operative retrograde urethrogram (Figure 3). One patient had a small diverticulum noted which was thought to be related to thin rectal mucosa. One patient developed a separate $2 \mathrm{~cm}$ stricture proximal to the rectal mucosa graft which required outpatient urethral dilation post-operatively. All patients were voiding without catheter at most recent follow-up. Subjectively, all four patients with prior BMGs stated preference for the rectal mucosal graft due to decreased post-operative discomfort from the graft site.

\section{Discussion}

Rectal mucosal graft substitution urethroplasty appears to be feasible in the management of urethral strictures in which buccal mucosal grafting is not an alternative. With greater than a year of follow-up in the five patients who underwent rectal mucosal grafting, all urethras remained patent and patients without a catheter at last follow-up. Mid and long term results are not yet available; however, this appears to be a promising alternative in patients with complicated urethral stricture disease.

Complications of TEM are rare and include pain or infection at the resection site, fecal incontinence, fistula, and rectal perforation (6-8). BMGs can be associated with prolonged mouth pain and risk of infection along with graft site contraction. In the current study, all four patients with prior BMGs stated that they would prefer a repeat rectal mucosal graft due to the lack of post-operative discomfort from the buccal harvest site $(9,10)$. While we do not suggest that this should drive surgical decision making, it certainly may contribute in patients concerned about the postoperative morbidity of buccal harvest.

Widespread dissemination of rectal mucosal graft substitution urethroplasty may be limited in part by the

Table 1 Patient and graft characteristics

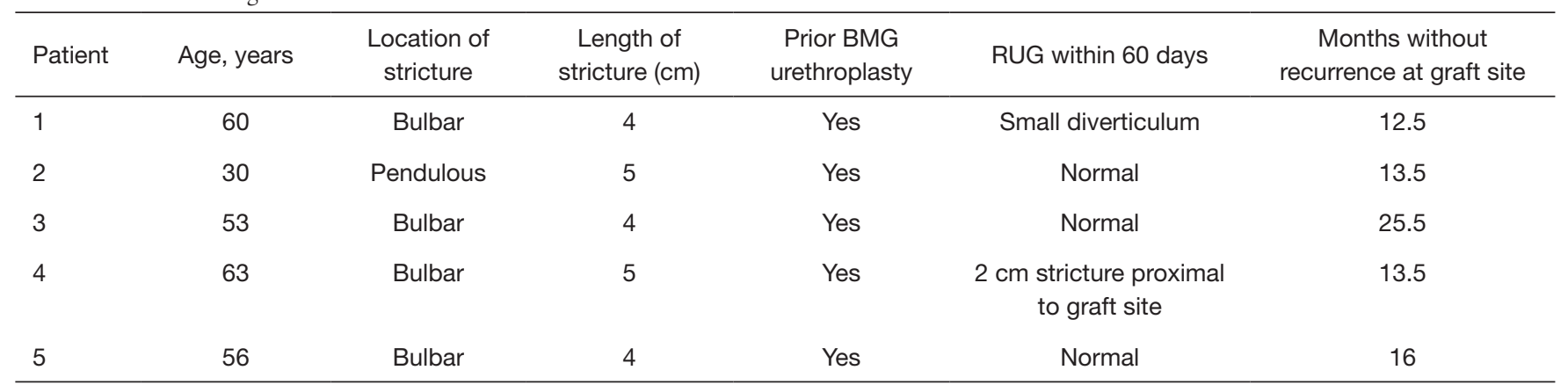

BMG, buccal mucosal graft. 

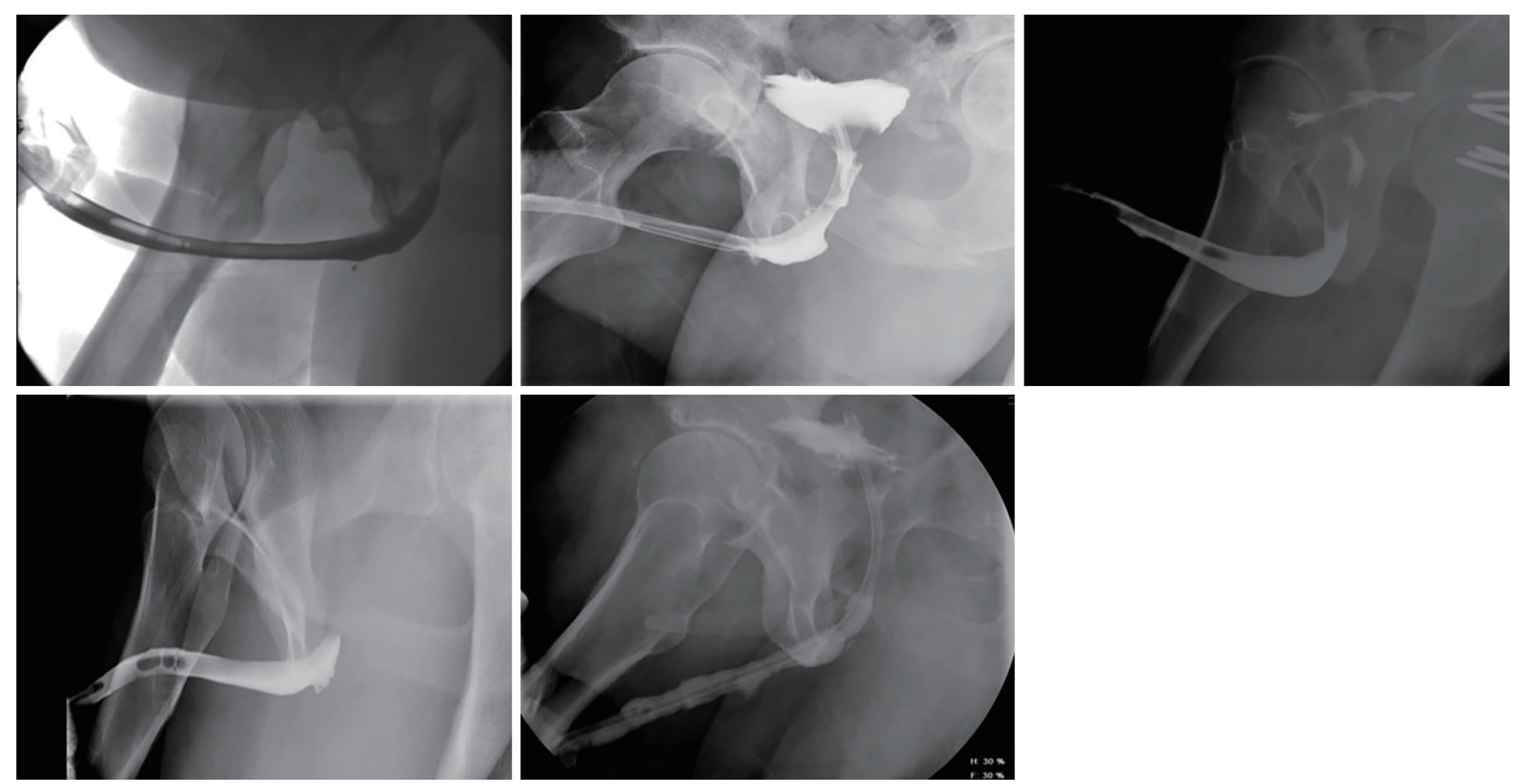

Figure 3 Post-operative retrograde urethrogram.

presence of colorectal surgeons trained in use of the TEM rectoscope for harvest of rectal grafts, although its dissemination and availability for the treatment of rectal pathology has become more common. The TEM rectoscope is an expensive technology for a hospital not already using it for colorectal operations. Additionally, there is a surgical learning curve is estimated between 14 and 24 cases $(11,12)$.

Multiple observations were made while performing out first rectal mucosal graft substitution urethroplasties. Firstly, the rectal mucosa graft itself is thinner and appears more prone toward forming diverticula without adequate tissue support. Additionally, as it is harvested with electrocautery, it required increased tailoring and trimming compared with BMGs. Finally, as the rectal mucosal graft was unable to be harvested at the same time as the perineal dissection to the urethra, there was significant time added for the conduct of these cases compared with our BMG urethroplasties.

The currently presented study has multiple limitations worth discussing. Firstly, it is a small study with limited follow-up. Additionally, we are dependent upon the patients following up in our clinic to know whether there were complications and if they continue voiding without difficulty to date. As the only tertiary care center in our state for Urologic care, we assume that they would return to us with issues but cannot guarantee that. Finally, the study is retrospective and was conducted only as a feasibility study. Regardless, we feel that our findings certainly further our understanding of how rectal mucosal grafts can be used in patients with urethral strictures who have contraindications to buccal mucosal grafting.

\section{Conclusions}

Short term data demonstrate that in carefully selected patients with contraindications to BMG harvesting, rectal mucosal grafts can provide a feasible, safe option for patients with urethral stricture disease. Mid- and long-term results are needed to further establish the place of rectal mucosal graft substitution urethroplasty in the armamentarium of reconstructive Urologists prior to its widespread use; however, early results are promising. Future research will be required to best delineate which patients benefit most from rectal as opposed to BMG urethroplasty.

\section{Acknowledgements}

None. 


\section{Footnote}

Conflicts of Interest: The authors have no conflicts of interest to declare.

Ethical Statement: The Indiana University School of Medicine Institutional Review Board exempt status was granted for the conduct of the study. As this was performed as a retrospective review of outcomes, patients did not undergo a separate consent from the procedural consent prior to surgery.

\section{References}

1. Lebret T, Gobet F, Dallaserra M, et al. Free appendix mucosa urethroplasty. A preliminary experimental study of free grafts of digestive tract mucosa. Prog Urol 1994;4:240-7.

2. Lebret T, Gobet F, Dallaserra M, et al. Use of a digestive mucosal graft in urethroplasty. An experimental study-prospective utilization of the appendix mucosa. Eur Urol 1995;27:58-61.

3. Xu YM, Qiao Y, Sa YL, et al. 1-stage urethral reconstruction using colonic mucosa graft for the treatment of a long complex urethral stricture. J Urol 2004;171:220-3; discussion 223.

4. Xu YM, Qiao Y, Sa YL, et al. One-stage urethral reconstruction using colonic mucosa graft: an experimental and clinical study. World J Gastroenterol 2003;9:381-4.

Cite this article as: Monn MF, Waters JA, Mellon MJ. Use of rectal mucosal grafts in substitution urethroplasty: an early series. Transl Androl Urol 2018;7(6):907-911. doi: 10.21037/ tau.2018.10.12
5. Palmer DA, Marcello PW, Zinman LN, et al. Urethral Reconstruction with Rectal Mucosa Graft Onlay: A Novel, Minimally Invasive Technique. J Urol 2016;196:782-6.

6. Clancy C, Burke JP, Albert MR, et al. Transanal endoscopic microsurgery versus standard transanal excision for the removal of rectal neoplasms: a systematic review and meta-analysis. Dis Colon Rectum 2015;58:254-61.

7. Fenig Y, Khoury M, Schmilovitz-Weiss H, et al. The outcome of transanal endoscopic microsurgery in obese patients. Eur J Gastroenterol Hepatol 2018;30:113-7.

8. Kumar AS, Coralic J, Kelleher DC, et al. Complications of transanal endoscopic microsurgery are rare and minor: a single institution's analysis and comparison to existing data. Dis Colon Rectum 2013;56:295-300.

9. Akyuz M, Gunes M, Koca O, et al. Evaluation of intraoral complications of buccal mucosa graft in augmentation urethroplasty. Turk J Urol 2014;40:156-60.

10. Soave A, Dahlem R, Pinnschmidt HO, et al. Substitution Urethroplasty with Closure Versus Nonclosure of the Buccal Mucosa Graft Harvest Site: A Randomized Controlled Trial with a Detailed Analysis of Oral Pain and Morbidity. Eur Urol 2018;73:910-22.

11. Helewa RM, Rajaee AN, Raiche I, et al. The implementation of a transanal endoscopic microsurgery programme: initial experience with surgical performance. Colorectal Dis 2016;18:1057-62.

12. Lee L, Kelly J, Nassif GJ, et al. Establishing the learning curve of transanal minimally invasive surgery for local excision of rectal neoplasms. Surg Endosc 2018;32:1368-76. 